\title{
Urología antigua en el Reino de Valencia. Apogeo y declive
}

\author{
López Alcina E*, Pérez Albacete $\mathrm{M}^{* *}$, Canovas Ivorra JA*** \\ *Hospital General Universitario de Valencia. Secretario de la (AUCV). ${ }^{* *}$ Coordinador de la Oficina de Historia \\ de la Asociación Española de Urología (AEU). ***Hospital Virgen de los Lirios. Alcoy (Alicante).
}

Actas Urol Esp. 2007;31(3):197-204

\section{RESUMEN}

UROLOGÍA ANTIGUA EN EL REINO DE VALENCIA. APOGEO Y DECLIVE

Durante la segunda mitad del siglo XVI se desplaza el interés de los clínicos hacia las dolencias venereas y las carnosidades uretrales (estrecheces) en detrimento de los procesos litiásicos.

Son de especial relevancia para nosotros, en el periodo cumbre de las ciencias médicas en tierras valencianas, Miguel Juan Pascual quien en su obra "Morborum internorum" (1555) sintetiza magistralmente todo lo conocido sobre la sífilis en su época, su origen, diagnóstico y curación, Juan Calvo considerado uno de los mejores cirujanos del siglo XVI y Miguel de Leriza autor del "Tratado del modo de curar las carnosidades y callos de la vía de la orina".

$\mathrm{El}$ barroco fue una época de crisis general en España y en el Antiguo Reino de Valencia, especialmente patente en campos como la anatomía y la cirugía. La grave decadencia que sufrió durante estos años la producción científica valenciana resulta evidente. Ni un solo texto anatómico se publicó en la primera mitad del siglo XVII, mientras que en el terreno quirúrgico solo la "Recopilación de toda la teórica y prática de Cirugía", de Alonso Romano puede considerarse digna de mención.

La ilustración trajo consigo nuevos aires reformistas y en torno a las florecientes academias científicas, se congregó lo más selecto de la intelectualidad valenciana. Andres Piquer Arrufat es considerado la máxima figura de la medicina valenciana de este periodo.

No es hasta después de la guerra de independencia y el reinado de Fernando VII que falleció en 1833 cuando se sientan las bases de la moderna urología y su consolidación como especialidad a finales del XIX. Las figuras valencianas principales de este periodo son Miguel Mas y Soler, Alejandro Settier y sobre todo Rafael Mollá y Rodrigo.

Palabras clave: Historia de la medicina. Historia de la urología. Historia de Valencia. Urología antigua.

\section{ABSTRACT}

\section{ANCIENT UROLOGY IN THE KINGDOM OF VALENCIA. HEIGHT AND DECLIVITY}

During the second half of the XVIth century the interest of the clinicians moves towards the venereal ailments and the urethral carnosities (narrowness) to the detriment of the litiasic processes.

They are of special relevancy for us, in the period summit of the medical sciences in Valencian lands, Miguel Juan Pascual who in his work "Morborum internorum" (1555) there synthesizes everything known on the syphilis in this epoch, its origin, diagnosis and treatment, Juan Calvo considered one of the best surgeons of the XVIth century and Miguel de Leriza author of the "Tractatus of the way of treating the carnosities and corns of the route of the urine".

The baroque was an epoch of general crisis in Spain and in the ancient Kingdom of Valencia, specially clear in fields as the anatomy and the surgery. The serious decadence that suffered during these years the scientific Valencian production turns out to be evident. Not even an alone anatomical text was published in the first half of the XVIIth century, whereas in the surgical area the "Summary of everything about the theoretical and practice of Surgery", of Alonso Romano can be considered deigns of mention.

The illustration brought new reformists airs and around the flourishing scientific academies, there congregated the most select of the Valencian intellectuality. Andres Piquer Arrufat is considered to be the maximum figure of the Valencian medicine of this period.

Not even after the war of independence and the reign of Fernando VII who died in 1833 when there sit down the bases of the modern urology and the consolidation as speciality at the end of the XIXth. The Valencian principal figures of this period are Miguel Más y Soler, Alejandro Settier and especially Rafael Mollá y Rodrigo.

Keywords: History of medicine. History of urology. History of Valencia. Ancient urology. 
A partir de la segunda mitad del siglo XVI como consecuencia de la propagación de las dolencias venéreas y su complicación más temible, las carnosidades uretrales (estrecheces) se desplaza el interés de los clínicos a estos problemas en detrimento de los procesos litiásicos. Prueba de ello es la proliferación de cirujanos con especial dedicación a tratar esta patología entre los que destacan, médicos y empíricos valencianos ${ }^{1}$. Es el caso del castellonense Miguel Juan Pascual que ocupó la Cátedra de Cirugía de la Universidad de Valencia de 1548 a 1561, ciudad donde publicó "Práctica copiosa de lo necesario para el cirujano" (1537) traducción comentada al castellano del tratado quirúrgico de Giovanni da Vigo, que fue reeditada en cinco ocasiones hasta el siglo XVIII y "Morborum internorum" (Fig. 1) impreso en Valencia por Juan Mey (1555), tratado de medicina práctica donde entre otros temas, describe el aparato urinario, la supresión de la orina, los cálculos de riñones y vejiga y la hematuria ${ }^{2}$. Este tratado fue uno de los más difundidos en Europa a través de seis reediciones durante el siglo XVI y cuatro durante el XVII (la última en 1664). Destaca su capitulo sobre el morbo gálico "De morbo quodum qui vulgo apud nos gallicus apellatur" en el que resume todo lo conocido de la enfermedad, su origen, diagnóstico y curación. El autor recomienda en relación con la enfermedad litiásica, la utilización de medicamentos que rompan la piedra junto con otros que lubriquen y dilaten, cuando el cálculo ha descendido a los uréteres. Cita las propiedades que deben tener los medicamentos que rompen la piedra tanto de riñón como de vejiga:

1. Deben aumentar la cantidad de orina.

2. Deben detenerse en los riñones y vejiga para que puedan ejercer su acción terapéutica.

3. Deben reforzar los riñones y la vejiga.

Seguidamente hace una relación de los diferentes medicamentos que rompen la piedra tanto de origen vegetal como animal y mineral. Por último compara los medicamentos simples y los compuestos llegando a la conclusión, como Galeno, de que los medicamentos compuestos son más seguros que los simples en el tratamiento de la litiasis urinaria.

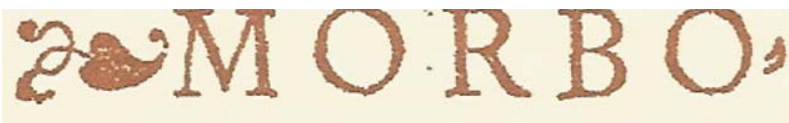

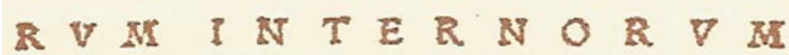
feré omnium, es gropundam externorm cur ts

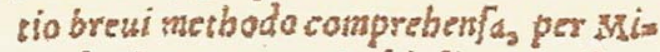
chä̈lem Lounnem $\mathrm{P}$ of chalium Medis cince profefforem Valentinum.

Huic difputatio gurdsm medic arceßit.

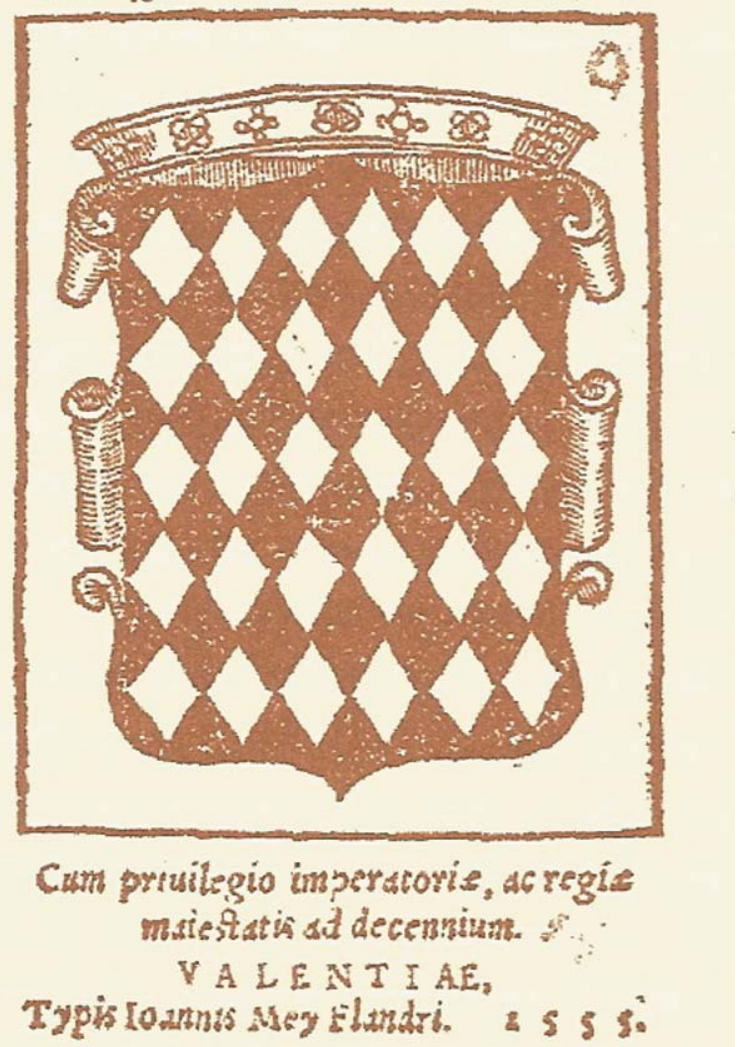

FIGURA 1. Portada de Morborum internorum. Miguel Juan Pascual. Editado en Valencia por Mey en 1555.

En relación con la abolición o retención de orina la describe como la situación: "aquella cuando hay supresión de orina la cual acontece cuando para del todo y no sale nada", y comenta el caso de un paciente tratado por él en el que consiguió su curación.

En su práctica copiosa explica en el capitulo titulado Acerca del arte de hacer mear con instrumentos que: "la orina retenida en la vejiga fuerza alguna vez al médico a que la saque con instrumento cañonado y corvo de cobre o de plata por la verga y manda que se haga esta obra cuando no puede mear por ser el camino de la orina cerrado de piedra o de sangre cuajada y también detiene 
muchas veces la orina con una apostemación pequeña y no sólo en los hombres, pero aún en las mujeres suele esto venir."

Otra de sus grandes obras "Praxis Médica" fue muy apreciada en su tiempo en varios países europeos, lo que explica que alcanzara once ediciones en poco más de un siglo. A partir de la cuarta (1579) fue impresa con los Scholia que redactó el médico setabense Pedro Pablo Pereda. Además su capítulo sobre la sífilis fue reproducido en la famosa colección de obras venereológicas del célebre médico italiano Luigi Luvigini ${ }^{3}$.

Otro valenciano, Juan Calvo fue uno de los mejores cirujanos del siglo XVI. Tras una estancia en la Universidad de Montpellier, se doctoró en Valencia donde fue discípulo de Collado y profesor de Cirugía desde 1568. Fundó una academia privada de Cirugía que era muy concurrida, donde asistían cirujanos de los diversos reinos hispánicos y médicos que deseaban completar su formación.

Publicó uno de los mejores tratados quirúrgicos del siglo "Cirugia Universal y particular del cuerpo humano" (Figs. 2 y 3), en dos tomos editados en 1580 y 1592 (se reeditó en castellano en 10 ocasiones y dos en francés). Lo redactó con destino a la enseñanza cuando llevaba doce años impartiendo clases de cirugía. En el primero habla, en lo que concierne a la urología, de los apostemas testiculares y de los tumores de todos los órganos contenidos entre las tres cavidades, entre los que incluye la hernia acuosa o hidrocele. En el segundo describe las carnosidades y el método de tratamiento con las candelillas. Manifiesta no ser partidario del empleo del instrumento cisorio para el tratamiento de las estrecheces de Francisco Díaz (acceso retrogrado) y Ambrosio Paré (acceso anterogrado), por las complicaciones que suele originar, en ocasiones con fatal resultado. Ideó algunas técnicas de interés, entre las que destacan al tratamiento quirúrgico de las varices y las fístulas, y la extirpación de los tumores malignos. La obra de Calvo también incluye un Tratado de anatomía de cierta amplitud y un Tratado de morbo gálico, que es el estudio más detenido de la clínica y el tratamiento de la sífilis de cuantos publicaron los cirujanos de la época ${ }^{4}$. También publicó en 1596 una traducción comentada al castellano del tratado quirúrgico de Guy de Chauliac.

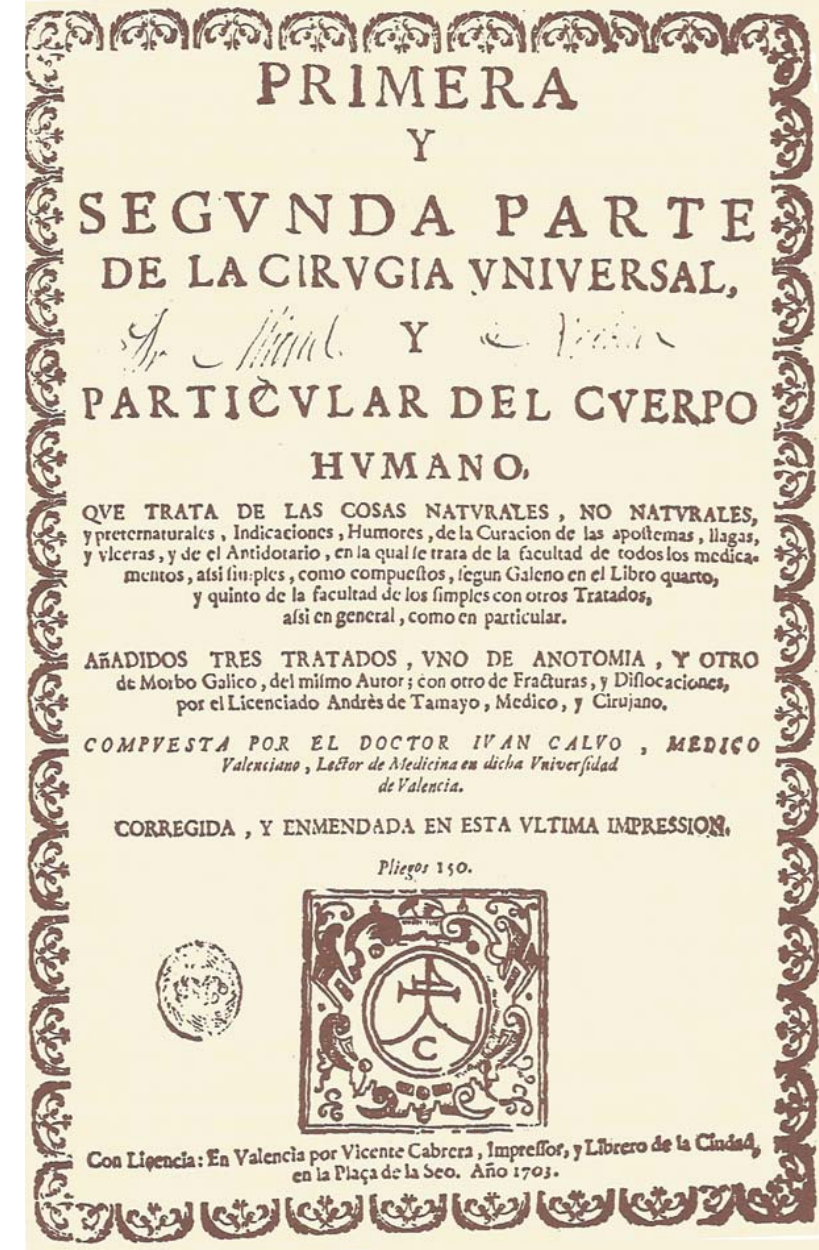

FIGURA 2. Portada de la edición de 1703 de la Cirugía de Juan Calvo. Publicada por primera vez en 1580 tuvo diez ediciones en castellano y dos en francés en poco más de un siglo.

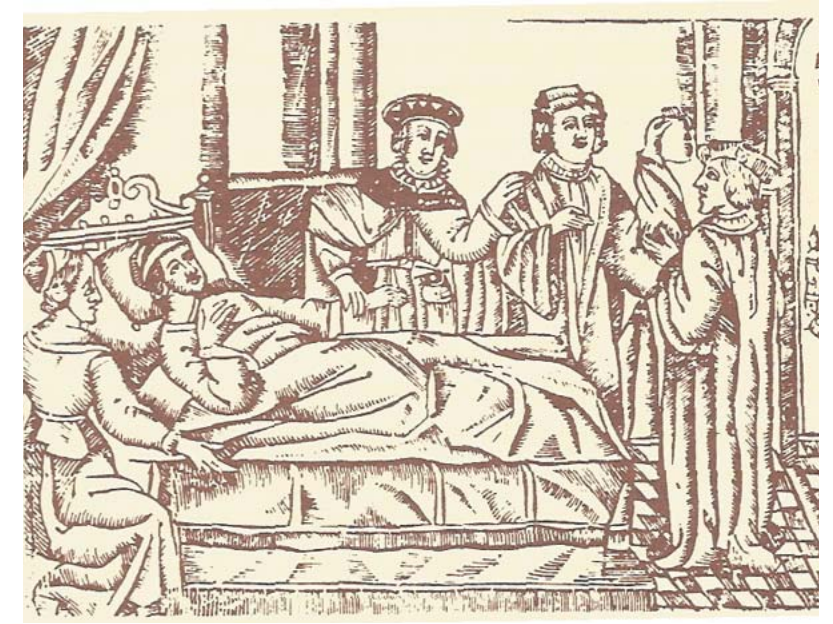

FIGURA 3. Uroscopia durante una consulta médica. Grabado de una obra del valenciano Juan Calvo (1596). 
Los datos biográficos de Miguel de Leriza son más bien escasos. Natural de Valencia donde estudió y ejerció la Medicina fue cirujano y oficial de la Santa Inquisición. Publicó en 1597 el "Tratado del modo de curar las carnosidades y callos de la via de la orina" (Fig. 4) donde resume y comenta distintos capítulos del Guido de Chauliac y del Tratado de Francisco Díaz. En relación del uso de las candelillas como método curativo, recomienda: "el uso de la geringación después de quitado el caústico, en el tratamiento de las carnosidades" - e indica el modo de proceder para introducir la candela - "estando el enfermo arrimado a la pared, derecho y con los pies

\section{TRATADO \\ DEL MODO DE CVRAR las carnofidades, y callos de la via de la orina.}

RECOPIL ADO POR MIGVEL de Leriza Círujano, Oficial de la Santa Inquificion de Valencia.

CONLAS ADVERTENCIAS para dicha cura neceforizas.

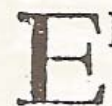
L prefente trarado, cl qual enfeña el modo de curar las carnofidades, y callos que eftan en la via de la orina, con. riene en sì quatro cofas para mayor cla. ridad.

La primera es, que la efcritura es nuyy fiel, y verdadera, por donde podra el artifice con muy buena conciencia exercitar to que en ella fe contiene, y con raucha confiança, y reguridad de la falud del en. fermo.

La fegunda, que los medicamentos, y remedios que trae para dichacura, fon los mejores, y mas ́reguros que hafta oy te han efcrito, ni hallado, y efto por la ex. periencia, y feliz fucefio que en las curas he tenibio por efpacio de mas de treinta

FIGURA 4. Página del Tratado del modo de curar las carnosidades y callos de la orina de Miguel de Leriza (1597). juntos, se tomará el miembro con la mano izquierda, encogiendo para ello el capullo, para que se descubra la vía de la orina y así con la mano derecha se tomará la candela untada en aceite, y la pondrá por la vía muy poco a poco".

En relación con la sintomatología urinaria define, entre otros, la estranguria: "cuando sale la orina poco a poco, o gota a gota y algunas veces acontece salir con ardor y escozor".

Por último Pedro Vives, natural de Orihuela y de profesión apotecari (boticario) debió conocer, durante su estancia en Valencia, las técnicas del Dr. Romano para el tratamiento de las carnosidades y molestias miccionales. En 1581 el concejo de Murcia solicitó al rey Felipe II autorización para que se desplazara a esta ciudad por considerarle "un experto en el tratamiento de las carnosidades y la pasión de orina" con objeto de aplicar la cura y enseñar su arte.

\section{LAS CIENCIAS UROLÓGICAS EN EL BARROCO VALENCIANO}

El barroco fue una época de crisis general en España. Se constató un acusado descenso de la población, recesión de la agricultura y tensiones sociales entre burguesía y nobleza. A lo largo de estos años decae la floreciente aportación de textos médicos del renacimiento.

La grave decadencia que sufrió durante estos años la producción científica valenciana resulta especialmente patente en campos como la anatomía y la cirugía. Se reeditaron las obras escritas en periodos anteriores, siendo las aportaciones originales a la literatura médica escasísimas. Ni un solo texto anatómico se publicó en la primera mitad del siglo, mientras que en el terreno quirúrgico sólo la "Recopilación de toda la teórica y práctica de Cirugia", de Alonso Romano (Fig. 5) puede considerarse digna de mención ${ }^{5}$. Nacido en Valencia, donde estudio Medicina y Cirugía. Fue médico de la cárcel de la corte y llegó a ser cirujano de Cámara del rey Felipe III. Escribió su obra cumbre en 1617 y fue reeditada en siete ocasiones, la última en 1647. Su contenido contrasta con el gran tratado quirúrgico que Juan Calvo había publicado medio siglo antes, ya que se limita a redactar un escueto y pobre manual, estructurado en preguntas y respuestas, y destinado al nivel más modesto de los profesionales de 


\section{RECOPILACION \\ DE TODA LA THEOR I CA, Y
PRACTICA DE CIRUGIA, MUY importante para los practicantes de ella. \\ COMPUESTAPOR EL DOCTOR \\ Alonfo Romano, Valenciano, Familiar del Santo Oficio, Medico, y Cirujano de la Camaspa del Rey Nueftro Señor del Reyro, y de la Care cel Real de fus coste. \\ AORA NUEUAMENTE LLEUR añadido vn Tratado del modo de cusar carnofje dades, y callos de la via de la orina, de Miguel de Leriza, Cirujano, Oficial de la Santa Inquificion de $V_{2}$ lencia. \\ En Valencia: Por Beniro Macè, junco el Is- figue Colegio del Patriarca. Áro de s66s. \\ Acofize de Matbeo Regil, Mercader de Libmaig delante de la Dipratacioswa}

FIGURA 5. Recopilación de toda la teórica y práctica de la cirugía. Alonso Romano. Edición de 1665.

la cirugía. Habla del hidrocele en el tratado de tumores particulares y se refiere a diversas afecciones de riñón y vejiga.

Félix Rodriguez de Gilbau se lamenta en su obra "Chirurgicorum in Valentia et eius Regno miseria" (1677) de la miserable situación en que se hallaban los cirujanos en Valencia y su reino. Oriundo de Valencia ocupó la cátedra de "herbes" (simples) y después la de "práctica" desde 1656 hasta su jubilación en 1681. Falleció en 1693. Como resultado de su dilatada experiencia médica tanto docente como práctica publicó en 1671 "Praxis médica Valentina", considerado uno de los mejores compendios de medicina práctica de su época. La exposición es muy clara y sistemática, destacando la excelente descripción de los fenómenos clínicos y la ponderación de las recomendaciones dietéticas y medicamentosas. Sigue la ordenación tradicional de patologías (a capite ad calces) en 3 partes (afecciones cefálicas, torácicas y abdominales); en esta última hace referencia a la litiasis en general y a las alteraciones de la micción. Se reeditó en 2 ocasiones (1681 y 1687) y fue durante muchos años libro de enseñanza en la Universidad de Valencia por su exposición metódica. Andrés Piquer lo cita el siglo siguiente por la claridad de sus descripciones clínicas $^{6}$.

\section{LAS CIENCIAS UROLÓGICAS EN LA ILUSTRACIÓN VALENCIANA}

La abolición de los fueros del Reino de Valencia (1707) situó en un nuevo marco político el desarrollo de la actividad científica valenciana. Este nuevo status quo, supuso por un lado una mayor dependencia externa, agravada por la práctica ausencia de instituciones médicas y científicas de promoción estatal semejantes a las que si se fundaron en Madrid, Barcelona o Cádiz ${ }^{7}$. Por otro lado, la nueva situación determinó, casi por obligación, un papel preponderante de la Universidad de Valencia, que sufrió una profunda renovación de sus estructuras, animada por los nuevos aires reformistas y en torno a las florecientes academias científicas, donde se congregaba lo más selecto de la intelectualidad valenciana $^{8}$.

Esta nueva mentalidad aperturista se constata en "Carta Filosófica Médico-Chymica", obra escrita por el médico valenciano Juan de Cabriada (1665-1714), y que, en opinión del historiador J.M. López Piñero, es el autentico documento fundacional de la renovación científica y cultural española. En un párrafo de dicha obra se denuncia el marasmo intelectual reinante en la época: "Es lastimosa, aún vergonzosa cosa, que como si fuéramos indios, hayamos de ser los últimos en recibir las noticias y luces públicas esparcidas por Europa"

En este ambiente surgen los llamados novatores, calificativo despectivo utilizado por los tradicionalistas para tildar a los partidarios de la ciencia y la filosofia moderna basada en la razón. Baguer y Oliver oriundo de Valencia, obtuvo en 1700 , el grado de Doctor en medicina en la Universidad de esta ciudad, donde llegó a ser Catedrático extraordinario. En su obra "Floresta de dissertaciones histórico-medicas, chymico- 
galénicas, methodico-practicas" publicada en Valencia en 1741, se refiere a la litogénesis cuando escribe que: "el cálculo renal se forma, cría y endurece un ácido exaltado de primeras vías, el que encontrándose con las partículas alcalinas, volátiles de la orina, y puestas en movimiento por la putrefacción, forman su competencia, y esta termina en la coagulación de unas, y otras partes, alas que se arriman algunas sales térreas, y otros áthomos; y de la unión, y coherencia de todos, resulta la piedra o cuerpo duro". Para la curación de la urolitiasis propone:

1. Quitar el dolor mediante ungüentos, o preparados orales a base de aceite de escorpión, altea, malva y violeta.

2. Sosegar la inflamación.

3. Demoler la piedra mediante un preparado al que llama bálsamo nefrítico.

4. y por último: "Restituir a su natural crasis el espíritu congenital o fermentativo de los riñones"

En el tomo II se ocupa de la estranguria y disuria, así como de la supresión de la orina: "que puede ser superior o inferior"

También Pascual Virrey Mangue nacido en Valencia en 1698, se formó como médico en la Universidad de esta ciudad, doctorándose en 1723. Opositó aunque sin éxito, a varias cátedras, así como a una plaza del Hospital General. Describe diversas afecciones urogenitales en su "Tirocinio práctico médico chimico-galénico" editado en 1737. Publicó en 1743 un Manual de Cirugía práctica, en dos volúmenes, único texto quirúrgico digno de mención redactado en la Valencia de la época. Esta estructurado en tres apartados: tumores, heridas y ulceras. Fue reeditado en dos ocasiones lo que demuestra que alcanzo cierta difusión e influencia. Sin embargo este manual no puede compararse a las rigurosas y actualizadas obras que publicaron poco después los profesores de los Reales Colegios de Cirugía, de Cádiz, Barcelona y Madrid.

Andres Piquer Arrufat (Fig. 6.) es considerado por J.M. López Piñero ${ }^{9}$, la máxima figura de la medicina valenciana de la ilustración. Se trasladó a Valencia en 1727 donde estudió Filosofía y Medicina graduándose en 1734. En 1741 entabló relación con Gregorio Mayans quien influyó en él

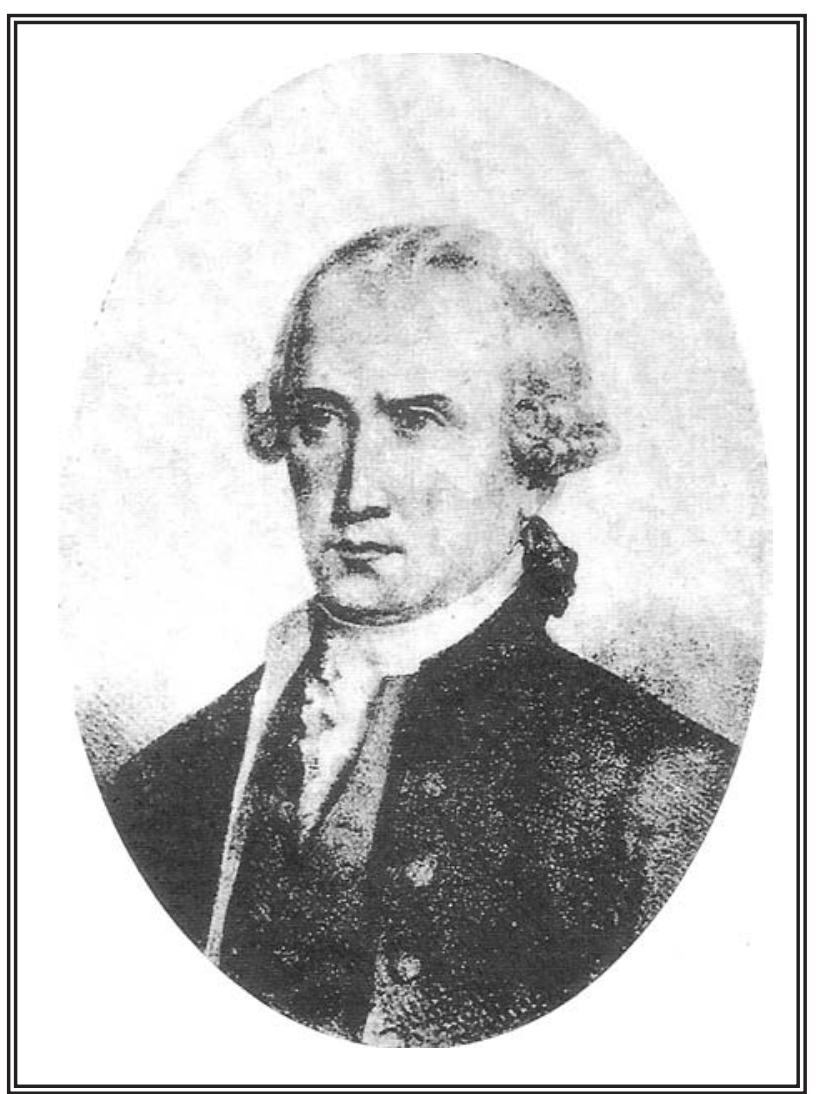

FIGURA 6. Andres Piquer. Máxima figura de la medicina valenciana de la ilustración.

sobre todo en su formación humanística y filosófica. Fue Catedrático de Anatomía y médico titular del Hospital General de Valencia desde 1742. En 1751 fue nombrado médico de cámara supernumerario de Fernando VI y trató a diferentes personalidades de la corte. Falleció en Madrid en 1772. Autor de gran producción científica, se considera su obra cumbre el "Tratado de las calenturas" impreso en Valencia 1751, que fue reimpresa en castellano en 5 ocasiones, y dos en francés, lo que da idea de su importante difusión. En otra de sus obras "Ad Tyrones" (1758), se refiere al tratamiento necesario para la expulsión de cálculos. En "Praxis médica. Ad usum Scholae Valentinae" (1762) dedica el capitulo XVI a De Morbis renum et vesicae donde se refiere a diversos síntomas urinarios y en relación con el mal de piedra concluye que: "la única solución al problema pasa por su expulsión al exterior. Para eso se debe ablandar y dilatar la vía y mover la naturaleza para arrojar al enemigo". También comenta que el mejor remedio para los 
cálculos es su prevención mediante: "la abstinencia de muchos y crasos alimentos, el uso frecuente de aceites..., la ingesta de agua fría que aumenta el vigor, la supresión total del vino y la realización de ejercicios moderados de cuerpo y mente". Considera a la litotomía una operación "peligrosa $e$ indecisa" además de que "arrojado [un calculo], producen otro". Esta obra fue reimpresa tras su muerte en tres ocasiones en España, en Amsterdam en 1775 y en Venecia en 1776, lo que da muestras de su influencia en la medicina de la época. Praxis médica junto al Tratado de las calenturas constituyeron una síntesis de medicina clínica doctrinal, no sólo utilizada en la Universidad de Valencia sino en otras españolas y extranjeras.

\section{UROLOGÍA VALENCLANA EN EL SIGLO XIX. ORÍGENES DE LA UROLOGÍA MODERNA}

La trayectoria que la actividad médica valenciana hubiera podido tener en la línea renovadora del siglo XVIII quedó truncada ante la crisis general de la España ilustrada de primeros del XIX, que culminó con la Guerra de la Independencia (1808-1814). La adversa situación política y socioeconómica junto a las reformas uniformadoras establecidas que perduraron durante el reinado de Fernando VII (1814-1833) perjudicó muy gravemente el cultivo de la ciencia en territorio valenciano. La Facultad de Medicina de Valencia fue suprimida en 1799 tras siglos de prolífica actividad en un intento de reorganización centralista que limitó la enseñanza a los Reales Colegios de Cirugía de Madrid, Barcelona y Cádiz.

El colapso de la medicina valenciana durante este primer tercio del siglo resulta patente incluso en el número de publicaciones de tema médico procedentes de las imprentas locales. Tal y como apunta López Piñero, entre 1731 y 1800, estas habían publicado 142 libros y folletos médicos, es decir, una media de 2 anuales Desde 1801 hasta 1833 solamente se editaron 50, lo que equivale a una media de 1,5 por año.

No resulta extraño que, durante estos años, Valencia no solamente perdiera la posición destacada que había tenido desde el Renacimiento dentro del panorama medico-quirúrgico y anatómico español, sino que, por primera vez desde entonces quedara desconectada de las corrientes centrales del saber científico-médico europeo.

No es hasta 1845, con la reforma del Ministro Pidal, que la Facultad de Medicina de Valencia, tras varios intentos en años precedentes, recupera la docencia correspondiente al título unificado de "Licenciado en Medicina y Cirugía". Ya durante la segunda mitad del XIX cabe destacar en el campo de las enfermedades genitourinarias la figura de Miguel Mas y Soler (18471902). Nacido en la localidad alicantina de Monovar obtuvo en 1878 plaza de profesor de Patología Quirúrgica en la Universidad de Valencia. A partir de ese momento llevó a cabo intervenciones innovadoras. En 1882 ingresó en la Academia de Medicina de Valencia con un discurso que verso sobre la uretrectomía. Presentó en el Congreso de Ciencias Medicas celebrado en Barcelona en 1888 dos nefrectomías por pionefrosis vía transperitoneal. En una de ellas llego a diagnóstico por punción percutánea. Relevantes fueron también las aportaciones de Alejandro Settier (1858-1915), oriundo de Valencia donde se licenció en Medicina y Cirugía. Completó su formación con Guyon en Paris. En 1887 dirige en Madrid la primera revista especializada "Gaceta de enfermedades de los órganos genitourinarios" (Fig. 7) y publica "Estrecheces de la uretra" libro donde aborda el diagnostico y tratamiento de la estenosis uretral de la época. Pero la figura principal y verdadero impulsor del desarrollo y consolidación de la Urología valenciana y española en el siglo XIX es Rafael Mollá y Rodrigo (18621930). Natural de Vinalesa, población cercana a Valencia, respaldó la creación de una Cátedra de Urología y fue presidente de la recientemente creada Asociación Española de Urología (AEU). Difundió la especialidad como director de varias revistas urológicas y fue autor de multitud de artículos y libros ${ }^{10}$. Fundó entre otras, la más prestigiosa revista valenciana especializada en la materia en la época, la Revista de Cirugía General y Genitourinaria (Fig. 8). Aún hoy en día, la AEU entrega una de sus becas, la de investigación clínica, en su recuerdo. 


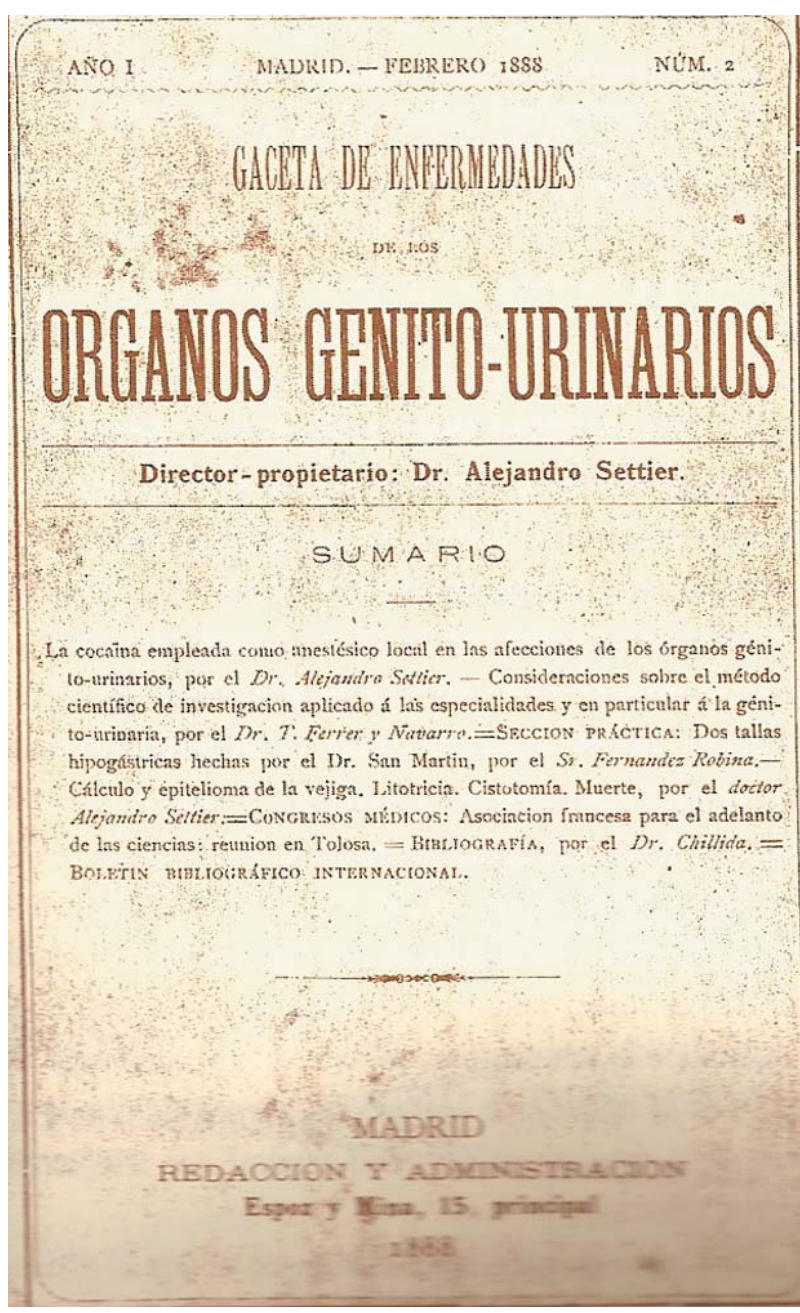

FIGURA 7. Gaceta de los órganos genitourinarios (1888). Primera revista monográfica española en el campo de la urología editada por el valenciano Alejandro Settier.

\section{REFERENCIAS}

1. García Ballester L.. La ciencia en Valencia después de la conquista. En: En torno al 750 aniversario de la conquista de Valencia. Valencia. Monografias del Consell Valencia de Cultura. 1989;239-254.

2. Pérez Albacete M. La urología en el Renacimiento y el Barroco. En: Historia Biográfica y Bibliográfica de la Urología Española. Madrid. Ediciones Edicomplet. 2000; 43-126.

3. Maganto Pavón E. Hitos en la Historia de la Urología. Barcelona. Ediciones Pulso. 1999.

4. López Terrada ML. La aparición del mal de siment en Valencia y su tratamiento. En: De hospitium, folls i malats: L'Hospital General de Valencia. Valencia, Ediciones del Ayuntamiento de Valencia. 2002;53-57.

5. López Piñero JM. Clásicos médicos valencianos del siglo XVI y XVII. Valencia. Ediciones de la Generalitat Valenciana, 1992.

6. Riera Palmero J. Historia de la Cirugía. Madrid. Editorial Médica Internacional. 1993.

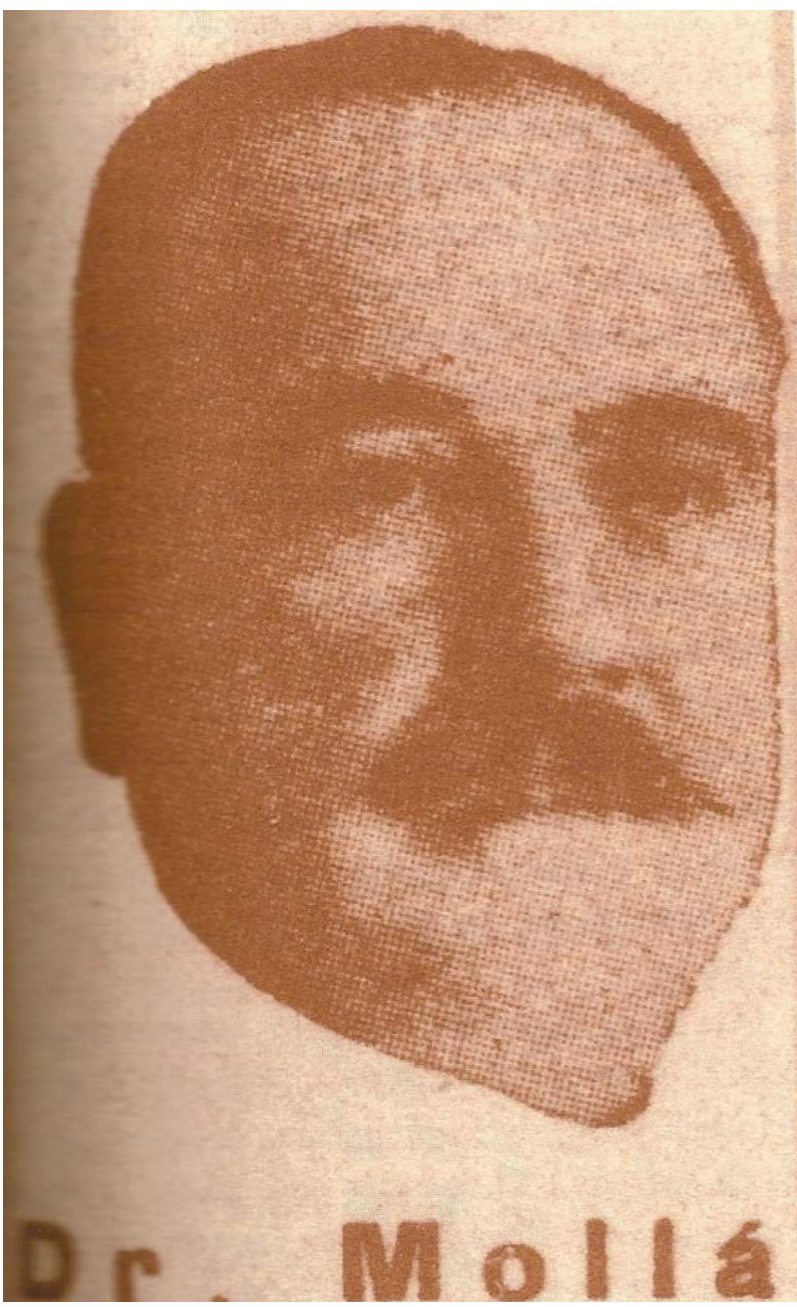

FIGURA 8. Rafael Molla y Rodrigo. Precursor de la urolo gía valenciana y española.

7. González F. Los hospitales a través de la historia y el arte. Barcelona. Ediciones Ars XXI. 2005

8. Furió A. Historia de Valencia. Valencia. Ediciones de la Universidad de Valencia. 1999.

9. López Piñero JM. Estudios sobre la profesión médica en la sociedad valenciana (1329-1898). Valencia, Ediciones del Ayuntamiento de Valencia. 1998.

10. Aguilar Bult, F. Rafael Mollá Rodrigo y la introducción de la Urología como especialidad en España. En: Actas del segundo congreso español de Historia de la Medicina, Salamanca, vol. I, 1965; pp. 219-224.

Correspondencia autor: Dr. E. López Alcina.

Servicio de Urología. Hospital General Universitario de Valencia

Avda. Tres cruces, s/n. 46014 Valencia

Tel.: 961972000

E-mail autor: eloalc@yahoo.es

Información artículo: Original - Historia de la Urología

Trabajo recibido: octubre 2006

Trabajo aceptado: enero 2007 